TRANSACTIONS OF THE

AMERICAN MATHEMATICAL SOCIETY

Volume 362, Number 1, January 2010, Pages 427-439

S 0002-9947(09)04948-4

Article electronically published on August 12, 2009

\title{
ALGEBRAIZATION OF BUNDLES ON NON-PROPER SCHEMES
}

\author{
VLADIMIR BARANOVSKY
}

\begin{abstract}
We study the algebraization problem for principal bundles with reductive structure groups on a non-proper formal scheme. When the formal scheme can be compactified by adding a closed subset of codimension at least 3 , we show that any such bundle admits an algebraization. For codimension 2 we provide a necessary and sufficient condition.
\end{abstract}

\section{INTRODUCTION}

This work is a contribution toward an algebraic understanding of the Uhlenbeck compactification. Recall, cf. [DK], that for a complex projective surface $S$ the moduli space $M_{n}$ of semistable vector bundles with fixed rank, determinant and $c_{2}=n$ is non-compact, but the union $U h l_{n}=\coprod_{s \geq 0} M_{n-s} \times S y m^{s} S$ can be given a topology of a compact space (since one deals with semistable bundles for $s \gg 0$ the space $M_{n-s}$ will be empty). We will call $U h l_{n}$ the Uhlenbeck moduli space, although sometimes this name is reserved for the closure of $M_{n}$ in $U h l_{n}$.

Some time ago, see e.g. [Li], BFG], FGK], the Uhlenbeck moduli space started to appear in algebraic geometry and the higher dimensional Langlands Program. For instance, it is a convenient tool for the study of higher versions of Hecke correspondences which modify a vector bundle on $S$ (more generally, a principal bundle) along a divisor, obtaining a new bundle. For several reasons, we would like to have the definition of $U h l_{n}$ as a "functor"; i.e. we want to be able to describe in geometric terms the set of maps $F(T)$ (actually, a category of maps) from any test scheme $T=\operatorname{Spec}(A)$ to $U h l_{n}$. First, that would allow us to define $U h l_{n}$ over any field $k$ and not to require stability. Second, in the study of the cohomology of $U h l_{n}$ and the action of Hecke correspondences on it, one needs to deal with the phenomenon of unexpected dimension of $U h l_{n}$. A possible approach involves defining a "derived moduli space" $D U h l_{n}$ in the sense of $[\mathrm{Lu}]$ which would amount to considering more general "spaces" $T$. Thus, defining $U h l_{n}$ as a functor is a necessary preliminary step to constructing $D U h l_{n}$.

Very roughly, it is expected that a map $T \rightarrow U h l_{n}$ should be described by a vector bundle $F$ on an open subset $U \subset T \times S$ such that its complement $Z$ is finite over $T$, a family $\xi$ of effective zero cycles on $S$ parameterized by $T$ plus an agreement condition between $\xi$ and $F$. Such a definition gives a "reasonable space" $U h l_{n}$ if it satisfies a criterion due to Artin, cf. [Ar], or its "derived" generalization proved in $[\mathrm{Lu}$. The most difficult part of Artin's criterion is the effectiveness condition: if $A$ is a complete noetherian local $k$-algebra with maximal ideal $\mathfrak{m}$ and $A_{p}=A / \mathfrak{m}^{p+1}$,

Received by the editors March 25, 2008.

2000 Mathematics Subject Classification. Primary 14D20.

This work was supported by a Sloan Research Fellowship.

(C)2009 American Mathematical Society 
one needs to show that $F(\operatorname{Spec}(A))=\varliminf F\left(\operatorname{Spec}\left(A_{p}\right)\right)$. Ignoring the family of zero cycles $\xi$ (as will be done in this paper), if $X=\operatorname{Spec}(A) \times_{k} S$ and $\widehat{X}$ is its formal completion along the fiber over the closed point of $\operatorname{Spec}(A)$, we try to find whether a bundle $\mathcal{F}$ on an open subset $\widehat{U} \subset \widehat{X}$ comes from a bundle $F$ on an open subset $U \subset X$. Such an $F$ is called an algebraization of $\mathcal{F}$.

In this paper we prove that, when $S$ has arbitrary dimension and $\widehat{U}$ has a complement of codimension $\geq 3$, algebraization always exists (for vector bundles and principal bundles over reductive groups). If $\widehat{U}$ has a complement of codimension $\geq 2$, then algebraization exists only under an additional condition (which, in the Uhlenbeck functor case, is guaranteed by the presence of $\xi$ ). For the codimension $\geq 3$ case the algebraization result is used in $\mathrm{Ba}$ to show that the corresponding functor of bundles "away from codimension 3" is an algebraic stack.

Earlier similar questions were studied for coherent sheaves on proper schemes by Grothendieck, see [EGAII], and in the case of Lefschetz type theorems by Grothendieck and Raynaud in SGA2 and [R]. Although these results do not apply to our case directly, our proof is based on the tools developed in [EGAIII] and SGA2].

In Section 2 we fix the notation, give examples illustrating some issues to be encountered, and prove algebraization results for vector bundles, summarized in Corollary 2.12. In Section 3 we formulate an algebraization criterion for principal bundles over reductive groups; see Theorem 3.1. Finally, Section 4 provides a categorical restatement of our results; see Theorem 4.2

\section{Algebraization for Vector Bundles}

2.1. Setup. We refer the reader to Expose III in [SGA2] regarding basic properties of depth and its relation to local cohomology. Let $S$ be an irreducible noetherian scheme of finite type over a field $k$. We will assume that $S$ is proper and satisfies Serre's $S_{2}$ condition: for any $s \in S$, $\operatorname{dept} h_{s} \mathcal{O}_{S} \geq \min \left(\operatorname{dim} \mathcal{O}_{S, s}, 2\right)$. Let $V \subset S$ be an open subset with closed complement of codimension $\geq 2$ in $S$ and $A$ a complete noetherian local $k$-algebra with residue field $K=A / \mathfrak{m}$ and associated graded $K$ algebra $\operatorname{gr}(A)=\bigoplus_{p \geq 0} g r_{p}(A)=\bigoplus_{p \geq 0} \mathfrak{m}^{p} / \mathfrak{m}^{p+1}$. Define $X=S \times_{k} \operatorname{Spec}(A)$ and

$$
X_{p}=S \times_{k} \operatorname{Spec}\left(A / \mathfrak{m}^{p+1}\right), \quad U_{p}=V \times_{k} \operatorname{Spec}\left(A / \mathfrak{m}^{p+1}\right), \quad p \geq 0 .
$$

Let $i_{p}: U_{p} \rightarrow X_{p}$ be the natural open embeddings. The completion $\widehat{X}$ of $X$ along $X_{0}$ may be viewed at the limit of $\left\{X_{p}\right\}_{p \geq 0}$; cf. Section 10.6 in [EGAI. The limit of $i_{p}$ gives an open formal subscheme $\widehat{i}: \widehat{U} \rightarrow \widehat{X}$. The ideal sheaf of $X_{0}$ in $X$ will be denoted by $\mathcal{J}_{X}$ and the closed subset $X_{0} \backslash U_{0}$ by $Z_{0}$. Finally, $f: X \rightarrow \operatorname{Spec}(A)$ is the natural proper projection and, for any $s \in \operatorname{Spec}(A), X_{s}$ stands for the fiber $f^{-1}(s)$.

Observe that $X$ may no longer satisfy the $S_{2}$ condition (since we made no depth assumptions on $A$ ). However, a particular case of Lemma 2 in $\mathrm{Ba}$ gives

Lemma 2.1. For any $x \in X$ with $f(x)=s$, depth $\mathcal{O}_{X, x} \geq \min \left(\operatorname{dim} \mathcal{O}_{X_{s}, x}, 2\right)$.

Consider a vector bundle $\mathcal{F}$ on $\widehat{U}$, i.e. a sequence of vector bundles $F_{p}$ on $U_{p}$ with isomorphisms

$$
\left.F_{p}\right|_{U_{p-1}} \simeq F_{p-1}, \quad p \geq 1 .
$$


Definition 2.2. We will say that a vector bundle $\mathcal{F}$ on $\widehat{U}$ admits an algebraization $(U, F)$ if there exists an open subset $U \subset X$ with $U \cap X_{0}=U_{0}$ and a vector bundle $F$ on $U$ such that $\mathcal{F}$ is isomorphic to the completion of $F$; i.e. for $\mathcal{J}_{U}=\left.\mathcal{J}_{X}\right|_{U}$ there exist isomorphisms $F_{p} \simeq F / \mathcal{J}_{U}^{p+1} F$ compatible with (2.1). In Section 3 we apply similar terminology to principal bundles.

Let $Z$ be the closed subset $X \backslash U$ and $i: U \hookrightarrow X$ the open embedding.

Lemma 2.3. Assume that $\operatorname{codim}_{X_{0}} Z_{0} \geq 2$ and an open subset $U \subset X$ satisfies $U \cap X_{0}=U_{0}$. For any $s \in \operatorname{Spec}(A)$, define $Z_{s}=Z \cap X_{s}$. Then codim $X_{X_{s}} Z_{s} \geq 2$ for all $s \in$ SpecA and $\operatorname{codim}_{X} Z \geq 2$.

Proof. Since $f$ is proper, the image $f\left(\bar{Z}_{s}\right)$ contains the unique closed point $s_{0} \in$ $\operatorname{Spec}(A)$. Therefore $\bar{Z}_{s} \cap X_{0} \subset Z_{0}$ is not empty. By semicontinuity of dimensions in the fibers we have $\operatorname{codim}_{X_{s}} Z_{s} \geq \operatorname{codim}_{X_{0}}\left(\bar{Z}_{s} \cap X_{0}\right) \geq \operatorname{codim}_{X_{0}} Z_{0}=2$. The second assertion of the lemma follows from the first.

Below we repeatedly use the following results.

Proposition 2.4. In the notation introduced above

(i) Completion along $X_{0}$ induces an equivalence between the category of coherent sheaves on $X$ and the category of coherent sheaves on the formal scheme $\widehat{X}$.

(ii) For any locally free sheaf $F$ (resp. $\left.F_{0}\right)$ on $U$ (resp. $\left.U_{0}\right)$ its direct image $i_{*} F\left(\right.$ resp $\left.\left(i_{0}\right)_{*} F_{0}\right)$ is coherent. If codim $X_{0} Z_{0} \geq 3$, then $R^{1}\left(i_{0}\right)_{*} F_{0}$ is also coherent.

(iii) Let $E$ be a coherent sheaf on $X$ and $\psi: E \rightarrow i_{*} i^{*} E$ the canonical morphism. Then $\psi$ is an isomorphism if and only if depth $h_{x} \geq 2$ for any point $x \in Z=X \backslash U$.

Proof. Part (i) follows from Corollary 5.1.6 in [EGAIII]. To check the coherence of $i_{*} F$, by Corollary VIII.2.3 in [SGA2] it suffices to check that $\operatorname{depth}_{x} F \geq 1$ for any point $x \in U$ such that $\overline{\{x\}} \cap Z$ has codimension 1 in $\overline{\{x\}}$. But Lemma 2.1 and local freeness of $F$ imply that any $x$ with $\operatorname{depth}_{x} F=0$ must be generic in its fiber, and Lemma 2.3 implies that $\overline{\{x\}} \cap Z$ would in fact have codimension 2 in $\overline{\{x\}}$. The same proof applies to $\left(i_{0}\right)_{*} F_{0}$. If $\operatorname{codim}_{X_{0}} Z_{0} \geq 3$ then the above argument can also by applied to $R^{1}\left(i_{0}\right)_{*} F_{0}$ once we show that $\operatorname{dept}_{x} F_{0} \geq 2$ for any $x \in U_{0}$ such that $\overline{\{x\}} \cap Z_{0}$ has codimension 1 in $\overline{\{x\}}$. But by $S_{2}$ condition $\operatorname{depth}_{x} F_{0} \leq 1$ can only hold for points $x$ of codimension $\leq 1$ in $U_{0}$, which would imply that $\overline{\{x\}} \cap Z_{0}$ has codimension $\geq 2$ in $\overline{\{x\}}$. This proves $(i i)$. Part (iii) is a particular case of Corollary II.3.5 in loc.cit.

2.2. Examples. The first example with $\operatorname{codim}_{X_{0}} Z_{0}=3$ and $K=k$ shows that one may not be able to take $U=U_{0} \times{ }_{k} \operatorname{Spec}(A)$.

Example 2.5. Take $S=X_{0}=\mathbb{P}^{3}$ with homogeneous coordinates $[x: y: z: w]$ and set $V=U_{0}=S \backslash[0: 0: 0: 1], A=k[[t]]$ (formal power series in $t$ ). Define vector bundles $F_{p}$ as kernels of

$$
\varphi_{p}: \mathcal{O}_{U_{p}}^{\oplus 3} \rightarrow \mathcal{O}(1)_{U_{p}} ; \quad\left(s_{1} \oplus s_{2} \oplus s_{3}\right) \mapsto s_{1} x+s_{2} y+s_{3}(z-t w) .
$$

Observe that $\varphi_{p}$ is surjective since $t$ is nilpotent on $U_{p}$ and $[0: 0: 0: 1] \notin U_{p}$. 
Lemma 2.6. The bundle $\mathcal{F}$ admits no algebraization $(U, F)$ with $U=U_{0} \times_{k}$ $\operatorname{Spec}(A)$.

Proof. Set $F$ to be the kernel of morphism $\varphi: \mathcal{O}^{\oplus 3} \rightarrow \mathcal{O}(1)$ of vector bundles on $U$, given by the same formula as for $\varphi_{p}$. By definition, $\varphi$ is not surjective only at $P=[0: 0: t: 1] \in U$, which projects to the generic point $\xi=\operatorname{Spec}\left(k\left[t^{-1}, t\right]\right) \in$ $\operatorname{Spec}(A)$. The specialization at $t=0$ is not in $U_{0}$, hence $P$ is closed in $U$ and $U \backslash P$ is an open subset containing $U_{0}$. Since on $U \backslash P$ we have the short exact sequence of locally free sheaves

$$
0 \rightarrow F \rightarrow \mathcal{O}^{\oplus 3} \rightarrow \mathcal{O}(1) \rightarrow 0,
$$

the restriction of $F$ to each $U_{p}$ is given by $F_{p}$, i.e. $\mathcal{F}$ is indeed the completion of $F$. On the other hand, $F$ is not locally free at $P$ : from $0 \rightarrow F \rightarrow \mathcal{O}_{U}^{\oplus 3} \rightarrow \mathcal{O}_{U} \rightarrow k_{P} \rightarrow 0$ we immediately get $\mathcal{E} x t^{1}\left(F, \mathcal{O}_{U}\right) \simeq \mathcal{E} x t^{3}\left(k_{P}, \mathcal{O}\right) \simeq k_{P}$ since the middle two terms are projective.

Suppose that $E$ is a locally free sheaf on $U$ with completion isomorphic to $\mathcal{F}$. We will see later in Proposition 2.11(ii) that in such a situation we must have $\widehat{i_{*} E} \simeq \widehat{i}_{*} \mathcal{F} \simeq \widehat{i_{*} F}$; hence by Proposition $2.4(\mathrm{i}), i_{*} F \simeq i_{*} E$, which contradicts $\mathcal{E} x t^{1}\left(F, \mathcal{O}_{U}\right) \neq 0$.

The second example illustrates that for $\operatorname{codim}_{X_{0}} Z_{0}=2$, a pair $(U, F)$ may not exist at all.

Example 2.7. Consider $A=k[[t]]$ and $S=X_{0}=\mathbb{P}^{2}$ with homogeneous coordinates $(x: y: z)$. Let $V=U_{0}=X_{0} \backslash P$, where $P=(0: 0: 1)$, and define a rank 2 bundle $F_{p}$ on $U_{p}=U_{0} \times_{k} \operatorname{Spec}\left(k[t] / t^{p+1}\right)$ as follows. The affine open subsets $U_{p}^{(x)}$, $U_{p}^{(y)}$ given by non-vanishing of $x$, resp. $y$, form a covering of $U_{p}$, and we can glue trivial rank 2 bundles on these open sets, using the transition function

$$
\left(\begin{array}{cc}
1 & \sum_{m=0}^{p}\left(\frac{t z^{2}}{x y}\right)^{m} \\
0 & 1
\end{array}\right)
$$

on $U_{p}^{(x)} \cap U_{p}^{(y)}$. Clearly $\left.F_{p}\right|_{U_{p-1}} \simeq F_{p-1}$ in a natural way, and we obtain a vector bundle $\mathcal{F}$ on $\widehat{U}$.

Lemma 2.8. There exists no vector bundle $F$ on $U=X \backslash Z$ with $\left.\widehat{F} \simeq \mathcal{F}\right|_{\widehat{U} \backslash\left(Z \cap U_{0}\right)}$ for any closed subset $Z \subset X$ such that $Z_{0} \subset\left(Z \cap X_{0}\right)$ and $\operatorname{codim}_{X_{0}}\left(Z \cap X_{0}\right) \geq 2$.

Proof. Suppose otherwise, and take the direct image of $F$ with respect to the open embedding $i: U \rightarrow X$. By Proposition 2.4 $i_{*} F$ is coherent and has depth $\geq 2$ at all codimension 2 points of $X$. Since modules of depth 2 over two-dimensional regular local rings are free by the Auslander-Buchsbaum formula, $i_{*} F$ will be locally free in codimension two. Therefore by shrinking $Z$ we can assume that $Z$ has codimension 3 in $X$, which in our case means that $Z$ is a finite set of points in $X_{0}$. Then the short exact sequence of sheaves on $X \backslash Z$

$$
0 \rightarrow F \stackrel{t^{p+1}}{\longrightarrow} F \rightarrow F_{p} \rightarrow 0
$$

(we identify $F_{p}$ with its direct image on $X \backslash Z$ abusing notation) gives a long exact sequence on $X$ :

$$
0 \rightarrow i_{*} F \stackrel{t^{p+1}}{\longrightarrow} i_{*} F \rightarrow i_{*} F_{p} \rightarrow R^{1} i_{*} F \stackrel{t^{p+1}}{\longrightarrow} R^{1} i_{*} F,
$$


where $R^{1} i_{*} F$ is coherent for the same reason as in Proposition 2.4(ii). Since $R^{1} i_{*} F$ is supported at the finite set $Z$ of closed points, it has finite length at each of them and the last arrow is zero for $p \geq p_{0}$. For such $p$ we can write $i_{*} F \rightarrow i_{*} F_{p} \rightarrow R^{1} i_{*} F \rightarrow 0$ which gives

$$
i_{*} F \otimes_{\mathcal{O}_{X}} k(P) \rightarrow i_{*} F_{p} \otimes_{\mathcal{O}_{X}} k(P) \rightarrow R^{1} i_{*} F \otimes_{\mathcal{O}_{X}} k(P) \rightarrow 0 .
$$

To prove the lemma it suffices to show that $\operatorname{dim}_{k} i_{*} F_{p} \otimes_{\mathcal{O}_{X}} k(P)$ is unbounded as $p \rightarrow \infty$.

To that end, replace $X_{0}$ with the affine open subset $\widetilde{X}_{0} \simeq \mathbb{A}^{2}$ given by the nonvanishing of $z$, with affine coordinates $u=\frac{x}{z}, v=\frac{y}{z}$. Set $W_{0}=U_{0} \cap \widetilde{X}_{0}$ and similarly for $\widetilde{X}_{p}, W_{p}, W_{p}^{(x)}$ and $W_{p}^{(y)}$. Then $\left.i_{*} F_{p}\right|_{\widetilde{X}_{p}}$ is the sheaf associated to $H^{0}\left(W_{p},\left.F_{p}\right|_{W_{p}}\right)$ viewed as a module over $A\left(\widetilde{X}_{p}\right)=k[u, v, t] / t^{p+1}$. By its definition, $F_{p}$ is an extension of $\mathcal{O}_{U_{p}}$ with $\mathcal{O}_{U_{p}}$ which leads to the long exact sequence

$$
0 \rightarrow H^{0}\left(W_{p}, \mathcal{O}_{W_{p}}\right) \rightarrow H^{0}\left(W_{p},\left.F_{p}\right|_{W_{p}}\right) \rightarrow H^{0}\left(W_{p}, \mathcal{O}_{W_{p}}\right) \rightarrow H^{1}\left(W_{p}, \mathcal{O}_{W_{p}}\right),
$$

where the last arrow sends the constant function 1 to the class of the extension. Let $M_{p}$ be the kernel of the last arrow. It suffices to show that $\operatorname{dim}_{k}\left(M_{p} /\langle u, v, t\rangle M_{p}\right)$ is unbounded. Computing $M_{p}$ via the affine covering $\left\{W_{p}^{(x)}, W_{p}^{(y)}\right\}$, we identify it with the kernel of

$$
k[u, v, t] / t^{p+1} \stackrel{\pi_{p} \circ \psi_{p}}{\longrightarrow} \frac{1}{u v} k\left[u^{-1}, v^{-1}, t\right] / t^{p+1},
$$

where $\psi_{p}$ is multiplication by $\sum_{l=0}^{p}\left(\frac{t}{u v}\right)^{l}$ (i.e. the upper right corner of the transition matrix in the definition of $F_{p}$ ) and $\pi_{p}$ is the natural projection

$$
k\left[u, u^{-1}, v, v^{-1}, t\right] / t^{p+1} \rightarrow \frac{1}{u v} k\left[u^{-1}, v^{-1}, t\right] / t^{p+1} .
$$

It follows that $M_{p}$ is generated by the monomials $t^{p}, t^{i} u^{p-i}, t^{i} v^{p-i}$ for $i=0, \ldots, p-$ 1 ; thus

$$
\operatorname{dim}_{k}\left(M_{p} /\langle u, v, t\rangle M_{p}\right)=2 p+1 \rightarrow \infty \quad \text { as } p \rightarrow \infty .
$$

Example 2.9 (Suggested to the author by V. Drinfeld). The bundle in the previous example has trivial determinant, but if we don't insist on that, there is a rank one example: glue two trivial line bundles on $U_{p}^{(x)}, U_{p}^{(y)}$ using the transition function $\sum_{m=0}^{p}\left(\frac{t z^{2}}{x y}\right)^{m}$. The resulting line bundle admits no algebraization since again $\operatorname{dim}_{k}\left(i_{p}\right)_{*} F_{p} \otimes_{\mathcal{O}_{X}} k(P)$ is not bounded as $p \rightarrow \infty$.

\subsection{Algebraization of vector bundles.}

Theorem 2.10. In the notation of Section 2.1,

(i) If codim ${ }_{X_{0}} Z_{0} \geq 3$, then $\mathcal{F}$ admits an algebraization.

(ii) If codim $_{X_{0}} Z_{0} \geq 2$ and the cokernel of the natural morphism $\left.\left(i_{p}\right)_{*} F_{p}\right|_{X_{p-1}} \rightarrow$ $\left(i_{p-1}\right)_{*} F_{p-1}$ is supported in codimension $\geq 3$ for all $p$ large enough, then $\mathcal{F}$ admits an algebraization.

(iii) In either of the two situations (codimension $\geq 3$ or codimension $\geq 2$ with the additional support assumption) the projective system $\left\{\left(i_{p}\right)_{*} F_{p}\right\}_{p \geq 0}$ satisfies the Mittag-Leffler condition, and the direct image $\widehat{i}_{*} \mathcal{F}$ is coherent and isomorphic to $\varliminf_{(}\left(i_{p}\right)_{*} F_{p}$. 
Proof. We split the proof of (i) and (ii) in a number of steps. Part (iii) will follow from Step 2.

Step 1. Suppose that $\widehat{i}_{*} \mathcal{F}$ is coherent. By Proposition 2.4(i) there exists a unique coherent sheaf $E$ on $X$ such that $\widehat{E} \simeq \widehat{i}_{*} \mathcal{F}$. The subset $U \subset X$ of points where $E$ is locally free is open and contains $U_{0}$ (e.g. by Nakayama's Lemma). Shrinking $U$ if necessary we can achieve $U \cap X_{0}=U_{0}$. Now set $F=\left.E\right|_{U}$.

Step 2. Therefore (i) and (ii) are reduced to showing that, under the conditions stated, $\widehat{i}_{*} \mathcal{F}$ is coherent. To that end we modify the argument of 0.13.7.7 in EGAIII which will also prove (iii). First, as in 0.13.7.2 of loc. cit., we choose injective resolutions $F_{k} \rightarrow L_{k}^{\bullet}$ such that $L_{k+1}^{\bullet} / \mathcal{J}_{U}^{k+1} L_{k+1}^{\bullet} \simeq L_{k}^{\bullet}$ and the natural filtrations by $\mathcal{J}_{U}^{n}(\ldots)$ agree with those on $F_{k}$. Each $\widehat{i}_{*}\left(L_{k}^{\bullet}\right)$ is a filtered complex and has a spectral sequence with the $E_{1}$ term given by

$$
E_{1}^{p q}=R^{p+q} \widehat{i}_{*}\left(\mathcal{J}_{U}^{p} F_{k} / \mathcal{J}_{U}^{p+1} F_{k}\right) .
$$

As in 0.13 .7 .3 of loc.cit. we pass to the limit as $k \rightarrow \infty$ and get a spectral sequence with

$$
E_{1}^{p, q}=R^{p+q} \widehat{i}_{*}\left(F_{p} / F_{p+1}\right) \simeq R^{p+q} \widehat{i}_{*}\left(F_{0}\right) \otimes_{K}\left(\mathfrak{m}^{p} / \mathfrak{m}^{p+1}\right)=R^{p+q} \widehat{i}_{*}\left(F_{0}\right) \otimes_{K} g r_{p}(A) .
$$

We are interested in the components

$$
E_{1}^{0}=\bigoplus_{p+q=0} E_{1}^{p, q}=\widehat{i}_{*}\left(F_{0}\right) \otimes_{K} \operatorname{gr}(A) ; \quad E_{1}^{1}=\bigoplus_{p+q=1} E_{1}^{p, q}=R^{1} \widehat{i}_{*}\left(F_{0}\right) \otimes_{K} \operatorname{gr}(A) .
$$

We would like to show that the spectral sequence converges at the $E^{0}=\bigoplus E^{p,-p}$ terms. Note that each $E_{k+1}^{1}=\bigoplus E_{k+1}^{p, 1-p}$ is a quotient of a subsheaf in $E_{k}^{1}$, while each $E_{k+1}^{0}$ is a subsheaf $E_{k}^{0}$ (since $E^{p,-1-p}$ terms are zero). Taking successive preimages of the boundaries in $E_{r-1}, E_{r-2}, \ldots, E_{1}$ we get a sequence of boundary subsheaves $B_{1} \subset B_{2} \subset B_{3} \subset \ldots \subset E_{1}^{1}$, and taking preimages of cycles in $E_{k}$ we get a sequence of cycle subsheaves $E_{1}^{0} \supset Z_{1} \supset Z_{2} \supset Z_{3} \supset \ldots$ By 0.13.7.6 in loc.cit. these are actually $\mathcal{O}_{X_{0}} \otimes_{K} g r(A)$-submodules.

Suppose that the sequence of cycles stabilizes; i.e. for some $r_{0}$ one has $Z_{r}=Z_{r_{0}}$ whenever $r \geq r_{0}$. Then by 0.13.7.4 in [EGAIII] the projective system $\left\{\widehat{i}_{*}\left(F_{k}\right)\right\}_{k \geq 0}$ satisfies the Mittag-Leffler condition and the associated graded of $\widehat{i}_{*}(\mathcal{F})$ is precisely $Z_{r_{0}} \subset \widehat{i}_{*}\left(F_{0}\right) \otimes_{K} \operatorname{gr}(A)$. But $\widehat{i}_{*}\left(F_{0}\right)$ is a coherent by Proposition 2.4 (ii), hence the subsheaf $\operatorname{gr}\left(\widehat{i}_{*} \mathcal{F}\right) \subset \widehat{i}_{*}\left(F_{0}\right) \otimes_{K} \operatorname{gr}(A)$ is a coherent $\mathcal{O}_{X_{0}} \otimes_{K} \operatorname{gr}(A)$-module by the noetherian property of $X_{0}$ and $A$. By loc.cit. 13.7.7.2, $\hat{i}_{*} \mathcal{F}$ is itself coherent on $\widehat{X}$. Also, $\widehat{i}_{*} \mathcal{F} \simeq \lim _{\longleftarrow}\left(i_{p}\right)_{*} F_{p}$ by 0.13.7.5.1 in loc.cit.

Step 3. Now the assertion of the theorem is reduced to showing that the sequence of cycles $Z_{1} \supset Z_{2} \supset \ldots$ stabilizes. By definition of $Z_{i}$ this is equivalent to saying that the higher differentials of the spectral seqence $d_{r}: E_{r}^{0} \rightarrow E_{r}^{1}$ become zero for $r \geq r_{0}$. That in turn is equivalent to saying that the sequence of boundaries $B_{1} \subset B_{2} \subset B_{3} \subset \ldots$ also stabilizes.

If $\operatorname{codim}_{X_{0}} Z_{0} \geq 3$ by Proposition 2.4(ii), $R^{1}\left(i_{0}\right)_{*} F_{0}$ is also coherent and $\left\{B_{r}\right\}_{r \geq 1}$ stabilizes by the noetherian property of $R^{1}\left(i_{0}\right)_{*} F_{0} \otimes_{K} \operatorname{gr}(A)$, which proves (i). If $\operatorname{codim}_{X_{0}} Z_{0} \geq 2$ we need to find a coherent subsheaf of $R^{1}\left(i_{0}\right)_{*} F_{0} \otimes_{K} \operatorname{gr}(A)$ containing $B_{r}$ for all $r \geq 1$. 
Step 4. At this point we reduced (ii) to showing that, under the assumptions stated, there exists a coherent subsheaf $G \subset R^{1}\left(i_{0}\right)_{*} F_{0}$ such that $B_{r} \subset G \otimes_{K} g r(A)$ for all $r$. By 0.11.2.2 in EGAIII for $r \geq p$, the term $B_{r}^{p, 1-p}$ is the image of the connecting homomorphism

$$
\widehat{i}_{*} F_{p} \rightarrow \widehat{i}_{*} F_{p-1} \stackrel{\rho_{p}}{\rightarrow} R^{1} \widehat{i}_{*} F_{0} \otimes_{K}\left(\mathfrak{m}^{p} / \mathfrak{m}^{p+1}\right)
$$

in the long exact sequence obtained by applying $R \hat{i}_{*}$ to the short exact sequence on $\widehat{U}$ :

$$
0 \rightarrow F_{0} \otimes_{K}\left(\mathfrak{m}^{p} / \mathfrak{m}^{p+1}\right) \rightarrow F_{p} \rightarrow F_{p-1} \rightarrow 0 .
$$

Observe that by our assumptions each $\operatorname{Im}\left(\rho_{p}\right)$ is coherent and supported in codimension $\geq 3$ for $p \gg 0$. Thefore we are done once we show that the subsheaf of $R^{1}\left(i_{0}\right)_{*} F_{0}$ formed by all sections with support in codimension $\geq 3$ is coherent whenever $\operatorname{codim}_{X_{0}} Z_{0} \geq 2$ and $F_{0}$ is locally free on $U_{0}$.

Step 5. Set $Q=\left(i_{0}\right)_{*} F_{0}$, a coherent sheaf on $X_{0}$ by Step 2. By the standard exact sequence we have $\mathcal{H}_{Z_{0}}^{2} Q=\left.R^{1}\left(i_{0}\right)_{*} Q\right|_{U_{0}}=R^{1}\left(i_{0}\right)_{*} F_{0}$, so it suffices to show that $\mathcal{H}_{\geq 3}^{0} \mathcal{H}_{Z_{0}}^{2} Q$ is coherent where $\mathcal{H}_{\geq 3}^{0}$ is the functor of sections supported in codimension $\geq 3$. Let $\mathcal{H}_{\geq 3}^{i}$ be the higher derived functors.

First, the standard spectral sequence for the composition of functors $R \mathcal{H}_{\geq 3}^{0}$, $R \mathcal{H}_{Z_{0}}^{0}$ has $E_{2}^{p, q}=\mathcal{H}_{\geq 3}^{p} \mathcal{H}_{Z_{0}}^{q} Q$. But $\mathcal{H}_{Z_{0}}^{i} Q=0$ for $i=0,1$ by Proposition 2.4(iii), so

$$
\mathcal{H}_{\geq 3}^{0} \mathcal{H}_{Z_{0}}^{2} Q \simeq \mathcal{H}_{\Phi}^{2} Q
$$

where the local cohomology $\mathcal{H}_{\Phi}^{2}$ has a family of supports

$$
\Phi=\left\{\text { all codim } \geq 3 \text { closed subsets in } Z_{0}\right\} .
$$

Step 6. To show that $\mathcal{H}_{\Phi}^{2} Q$ is coherent note that by [Ha2] the scheme $X_{0}$ has a dualizing complex $\omega$ of the form

$$
0 \rightarrow \mathcal{K}^{0} \rightarrow \ldots \rightarrow \mathcal{K}^{\operatorname{dim}_{K} X_{0}} \rightarrow 0
$$

with $\mathcal{K}^{i}=\bigoplus_{\text {dim }_{\mathcal{O}_{0}, x}=i} J(x)$ and each $J(x)$ is the direct image of the injective envelope of the residue field $k(x)$ with respect to the natural morphism $i^{x}$ : $\operatorname{Spec}\left(\mathcal{O}_{X_{0}, x}\right) \rightarrow X_{0}$. By definition of a dualizing complex, the double complex $\mathcal{K}^{p, q}=\mathcal{H o m}\left(\mathcal{H o m}\left(Q, \mathcal{K}^{-q}\right), \mathcal{K}^{p}\right)$ has a total complex quasi-isomorphic to $Q$. Moreover, by Proposition IV.2.1 and the remark on page 123 in [Ha2, the total complex is a flasque resolution of $Q$ and hence can be used to compute $\mathcal{H}_{\Phi}^{\bullet}(Q)$. This leads to a spectral sequence:

$$
E_{2}^{p, q}=\mathcal{E} x t_{\Phi}^{p}\left(\mathcal{E} x t^{-q}(Q, \omega), \omega\right) \Rightarrow \mathcal{H}_{\Phi}^{p+q}(Q),
$$

where $\mathcal{E} x t_{\Phi}^{p}=R^{p}\left(\Gamma_{\Phi} \circ \mathcal{H}\right.$ om $)$ and the $\mathcal{E} x t$ sheaves are understood in the sense of hypercohomology.

Only finitely many terms $E_{2}^{p, q}$ with $p+q=2$ will be non-trivial: since $\mathcal{K}^{q}$ are injective, the non-vanishing implies $0 \leq(-q) \leq \operatorname{dim}_{K} X_{0}$. Thus it suffices to show that $E_{2}^{p, 2-p}=\mathcal{E} x t_{\Phi}^{p}\left(\mathcal{E} x t^{p-2}(Q, \omega), \omega\right)$ is coherent for $p \geq 2$.

An important observation which we use below is that $\mathcal{K}^{p}$ has no sections supported in codimension $\geq p+1$.

Step 7. First observe that $\mathcal{E} x t_{\Phi}^{2}(G, \omega)=0$ for any quasi-coherent sheaf $G$ since $\mathcal{K}^{2}$ has no sections supported in codimension $\geq 3$ and hence no sections with support in $\Phi$. Hence we can assume that $p \geq 3$. 
We first claim that $\operatorname{codim}_{X_{0}} \operatorname{Supp}\left(\mathcal{E} x t^{p-2}(Q, \omega)\right)=d \geq p \geq 3$. In fact, let $x \in \operatorname{Supp}\left(\mathcal{E} x t^{p-2}(Q, \omega)\right)$ be a point with $\operatorname{dim} \mathcal{O}_{X_{0}, x}=d$. By local duality, cf. V.6 in [Ha2, the non-vanishing of the stalk $\mathcal{E} x t^{p-2}(Q, \omega)_{x}$ is equivalent to the non-vanishing of local cohomology $\mathcal{H}_{x}^{d+2-p}(Q)$, which implies $d+2-p \geq 0$ and $d \geq p-2 \geq 1$. If $d=1$, then $p=3$ and also $x \notin Z_{0}$; hence the stalk $Q_{x}$ is free. Thus $\mathcal{H}_{x}^{0}(\mathcal{O}) \neq 0$, contradicting the $S_{2}$ assumption. If $d \geq 2$, then applying the $S_{2}$ condition when $x \notin Z_{0}$ and Proposition 2.4(iii) when $x \in Z_{0}$, we actually have $d+2-p \geq 2$, so $d \geq p$ as required.

By primary decomposition, the coherent sheaf $\mathcal{E}_{x} t^{p-2}(Q, \omega)$ admits a finite filtration by coherent subsheaves such that all successive quotients have irreducible supports of codimension $\geq p$. By the standard long exact sequence for $\mathcal{E} x t_{\Phi}^{\bullet}(\cdot, \omega)$ is suffices to show that $\mathcal{E}_{x} t_{\Phi}^{p}(G, \omega)$ is coherent whenever $p \geq 3$ and $G$ is a coherent sheaf with irredicuble support $Y$ of codimension $\geq p$.

If $Y \nsubseteq Z_{0}$ for any $W$ in the family $\Phi$, the intersection $Y \cap W$ is not equal to $Y$ and therefore has codimension $\geq p+1$. But then $\mathcal{E} x t_{\Phi}^{p}(G, \omega)=0$, because any section $\rho$ of $\mathcal{H o m}\left(G, \mathcal{K}^{p}\right)$ representing a class in $\mathcal{E} x t_{\Phi}^{p}(G, \omega)$ has zero values since $\mathcal{K}^{p}$ has no sections supported in codimension $\geq p+1$. If $Y \subseteq Z_{0}$, then $Y$ is an element of $\Phi$ and $\mathcal{E} x t_{\Phi}^{p}(G, \omega) \simeq \mathcal{E} x t^{p}(G, \omega)$ since all sections of $\mathcal{H o m}\left(G, \mathcal{K}^{t}\right)$ have support in $\Phi$. But $\mathcal{E} x t^{p}(G, \omega)$ is coherent, which finishes the proof.

The converse to Theorem 2.10 can be formulated as follows.

Proposition 2.11. In the setting of Section 2.1, assume that $\mathcal{F}$ admits an algebraization $(U, F)$ and view each $F_{p}$ as a sheaf on $U$. Then

(i) The cokernel of $i_{*} F_{p} \rightarrow i_{*} F_{p-1}$ is supported in codimension $\geq 3$ for $p \gg 0$.

(ii) The isomorphism $\widehat{F} \simeq \mathcal{F}$ extends to direct images: $\widehat{i_{*} F} \simeq \widehat{i}_{*} \mathcal{F}$. In particular, $\widehat{i}_{*} \mathcal{F}$ is coherent.

Proof. To prove (i) observe that the cokernel of $i_{*} F_{p} \rightarrow i_{*} F_{p-1}$ is annihilated by $\mathcal{J}_{X}$, being a subsheaf of $R^{1} i_{*} F_{0} \otimes_{K} g r_{p}(A)$, and is therefore isomorphic to the cokernel of $\left.\left.i_{*} F_{p}\right|_{X_{0}} \rightarrow i_{*} F_{p-1}\right|_{X_{0}}$.

We will first show that the natural map $\left.i_{*} F_{p}\right|_{X_{0}} \rightarrow i_{*} F_{0}$ is an embedding of sheaves for all $p$. Considering the exact sequence

$$
\left.0 \rightarrow \mathcal{J}_{X}\left(i_{*} F_{p}\right) \rightarrow i_{*} F_{p} \rightarrow i_{*} F_{p}\right|_{X_{0}} \rightarrow 0
$$

and its map to the first terms of the sequence

$$
0 \rightarrow i_{*}\left(\mathcal{J}_{U} F_{p}\right) \rightarrow i_{*} F_{p} \rightarrow i_{*} F_{0} \rightarrow R^{1} i_{*}\left(\mathcal{J}_{U} F_{p}\right) \rightarrow \ldots,
$$

we see that $\left.i_{*} F_{p}\right|_{X_{0}} \rightarrow i_{*} F_{0}$ is an embedding precisely when the natural map $\mathcal{J}_{X}\left(i_{*} F_{p}\right) \rightarrow i_{*}\left(\mathcal{J}_{U} F_{p}\right)$ is an isomorphism. Observe that $i_{*} \mathcal{O}_{U}=\mathcal{O}_{X}$; hence $i_{*} \mathcal{J}_{U}$ is a sheaf of ideals in $\mathcal{O}_{X}$.

Using Lemma 2.1 and the Cohen-Macaulay assumption on $X_{0}$, we see that $\mathcal{H}_{Z}^{t} \mathcal{O}_{X}=\mathcal{H}_{Z_{0}}^{t} \mathcal{O}_{X_{0}}=0$ for $t=0,1$. By the short exact sequence $0 \rightarrow \mathcal{J}_{X} \rightarrow$ $\mathcal{O}_{X} \rightarrow \mathcal{O}_{X_{0}} \rightarrow 0$ we derive $\mathcal{H}_{Z}^{t} \mathcal{J}_{X}=0$ for $t=0,1$, and hence $\mathcal{J}_{X}=i_{*} \mathcal{J}_{U}$ by Proposition 2.4(iii). Then

$$
i_{*}\left(\mathcal{J}_{U} F_{p}\right)=\left(i_{*} \mathcal{J}_{U}\right)\left(i_{*} F_{p}\right)=\mathcal{J}_{X} i_{*} F_{p},
$$

as required. Similarly, $\left.i_{*} F\right|_{X_{0}} \rightarrow i_{*} F_{0}$ is an embedding. So for any $p \geq 1$ we have the embeddings

$$
\left.\left.\left.i_{*} F\right|_{X_{0}} \hookrightarrow i_{*} F_{p}\right|_{X_{0}} \hookrightarrow i_{*} F_{p-1}\right|_{X_{0}} \hookrightarrow i_{*} F_{0} .
$$


Consequently, the coherent sheaf $\mathcal{K}=\operatorname{Coker}\left(\left.i_{*}(F)\right|_{X_{0}} \rightarrow i_{*} F_{0}\right)$ has a decreasing filtration by images of $\left.i_{*} F_{p}\right|_{X_{0}}$, and each $\operatorname{Coker}\left(\left.\left.i_{*} F_{p}\right|_{X_{0}} \rightarrow i_{*} F_{p-1}\right|_{X_{0}}\right)$ is its successive quotient. But $\mathcal{K}$ is a coherent sheaf with $\operatorname{Supp}(\mathcal{K}) \subset Z_{0}$ and $Z_{0}$ has at most finitely many points of codimension 2 . Since for each point $x \in X_{0}$ of codimension 2 the localization $\mathcal{K}_{x}$ is a module of finite length, only finitely many successive quotients of the filtration of $\mathcal{K}$ can be non-trivial in codimension 2, which proves (i).

To prove (ii) first observe that $\widehat{i}_{*} \mathcal{F}$ and $E=i_{*} F$ are coherent by Theorem 2.10(iii) and Proposition 2.4(ii), respectively. By Proposition 2.4(i) we can find a sheaf $E^{\prime}$ such that $\widehat{E^{\prime}} \simeq \widehat{i}_{*} \mathcal{F}$. The isomorphism $\left.\widehat{E}\right|_{\widehat{U}} \simeq \mathcal{F}=\widehat{i}^{*} \widehat{i}_{*} \mathcal{F}$ extends uniquely to a morphism of sheaves $\widehat{\phi}: \widehat{E} \rightarrow \widehat{i}_{*} \mathcal{F}=\widehat{E}^{\prime}$. By Proposition 2.4(i), $\widehat{\phi}$ is the completion of a unique morphism $\phi: E \rightarrow E^{\prime}$ which by Corollary 10.8.14 in [EGAI] should be an isomorphism on an open subset $W$ containing $U_{0}$. Shrinking $W$ if necessary we can assume $W \subset U$. By Lemma 2.3, each point $x \in U \backslash W$ has codimension $\geq 2$ in its fiber; hence $\operatorname{depth}_{x} E \geq 2$ by Lemma 2.1. For $x \in X \backslash U$ we still have depth $h_{x} E \geq 2$ by Proposition 2.4(iii). Applying the same result to $j: W \hookrightarrow X$ instead of $U$ we see that $E=j_{*} j^{*} E$. By adjunction of $j^{*}$ and $j_{*}$ the isomorphism $\left(\left.\phi\right|_{W}\right)^{-1}: j^{*} E^{\prime} \rightarrow j^{*} E$ extends uniquely to a morphism $\psi: E^{\prime} \rightarrow$ $j_{*} j^{*} E=E$.

By construction, the composition $\psi \phi: E \rightarrow E$ restricts to identity on $W$; hence $\psi \phi=I d_{E}$, by the same adjunction. Similarly, the composition $\widehat{\phi} \widehat{\psi}=\widehat{E^{\prime}} \rightarrow \widehat{E^{\prime}}$ restricts to identity on $\widehat{U}$, and since $\widehat{E^{\prime}} \simeq \widehat{i}_{*} \mathcal{F}$, we must have $\widehat{\phi} \widehat{\psi}=I d_{\widehat{E^{\prime}}}$, so $\phi \psi=I d_{E^{\prime}}$ by Proposition 2.4(i). We have proved that $E=i_{*} F \simeq E^{\prime}$. Since $\widehat{E^{\prime}}=\widehat{i}_{*} \mathcal{F}$ we conclude that $\widehat{i_{*} F}=\widehat{i}_{*} \mathcal{F}$.

Corollary 2.12. The following conditions are equivalent:

(i) The cokernel of $\left.\left(i_{p}\right)_{*} F_{p}\right|_{X_{p-1}} \rightarrow\left(i_{p-1}\right)_{*} F_{p-1}$ is supported in codimension $\geq 3$ for $p \gg 0$.

(ii) The projective system $\left\{\hat{i}_{*} F_{p}\right\}_{p \geq 1}$ satisfies the Mittag-Leffler condition.

(iii) The direct image $\widehat{i}_{*} \mathcal{F}$ is coherent.

(iv) The bundle $\mathcal{F}$ admits an algebraization.

Proof. The implications (i) $\Rightarrow$ (ii) and (iii) $\Rightarrow$ (iv) are established in the proof of Theorem 2.10. The implication (iv) $\Rightarrow$ (i) is proved in Proposition 2.11, If the projective system $\left\{\widehat{i}_{*} F_{p}\right\}_{p \geq 1}$ satisfies the Mittag-Leffler condition, by 0.13.3.1 in EGAIII the natural map $\widehat{i}_{*} \mathcal{F} \rightarrow \lim \widehat{i}_{*} F_{p}$ is an isomorphism. By the MittagLeffler condition we can replace $\widehat{i}_{*} F_{p}$ by a system of subsheaves $G_{p} \subset \widehat{i}_{*} F_{p}$ so that the property $\widehat{i}_{*} \mathcal{F} \simeq \lim _{\longleftarrow} G_{p}$ still holds and $\left.G_{p}\right|_{X_{p-1}} \rightarrow G_{p-1}$ is surjective. Since each $G_{p}$ is coherent by the noetherian property of $X_{p}$, Proposition 10.11.3 in [EGAI] tells us that $\lim _{\longleftarrow} G_{p}$ is also coherent. Therefore, (ii) $\Rightarrow$ (iii).

Remark 2.13. Suppose that $X_{0}$ is a smooth projective surface over $K, \xi=k_{1} P_{1}+$ $\ldots+k_{l} P_{l}$ an effective zero cycle and that $F_{0}$ is a rank $n$ vector bundle on $U_{0}=$ $X_{0} \backslash\left\{P_{1}, \ldots, P_{l}\right\}$. The pair $\left(F_{0}, \xi_{0}\right)$ should define a point $\operatorname{Spec}(K) \rightarrow U h l_{n}$ of the Uhlenbeck functor. Assume that $(F, \xi): \operatorname{Spec}(A) \rightarrow U h l_{n}$ extends $\left(F_{0}, \xi_{0}\right)$. Then it is expected that $\operatorname{Coker}\left(i_{*} F \rightarrow i_{*} F_{0}\right)$ can be supported only at the points $P_{1}, \ldots, P_{l}$, with multiplicities bounded by $k_{1}, \ldots, k_{l}$, respectively. (In the differential geometry picture, cf. [DK], $\xi_{0}$ represents the singular part of a connection 
which may be smoothed out by $F$ but may not acquire any negative coefficients. Since the multiplicities of $\operatorname{Coker}\left(i_{*} F \rightarrow i_{*} F_{0}\right)$ measure the local change of $c_{2}$, one obtains the bound mentioned.) But the proof of Proposition 2.11 shows that the multiplicities of $\operatorname{Coker}\left(i_{*} F \rightarrow i_{*} F_{0}\right)$ give an upper bound for the total sum, over all $p$, of similar multiplicities for $\operatorname{Coker}\left(\left.\left(i_{p}\right)_{*} F_{p}\right|_{X_{p-1}} \rightarrow\left(i_{p-1}\right)_{*} F_{p-1}\right)$. Hence the condition of Corollary 2.12(i) is rather natural from the point of view of Uhlenbeck spaces.

\section{Algebraization of PRINCIPAL BUndLeS}

Let $G$ be an affine algebraic group over $k$. We keep the notation of Section 2.1 and consider left principal $G$-bundles which are locally trivial in fppf topology. For such a $G$-bundle $P$ (over $\widehat{U}$ or an open subset $U \subset X$ ) and any scheme $Y$ over $k$ with left $G$-action, denote by $P_{Y}=G \backslash\left(Y \times_{k} P\right)$ the associated fiber bundle, i.e. the quotient by the left diagonal action of $G$. For instance, when $\rho: G \rightarrow H$ is a homomorphism of linear algebraic groups over $k$ we can consider a left $G$-action on $H$ given by $g \cdot h=h \rho(g)^{-1}$, and then $P_{H}$ is simply the principal $H$-bundle induced via $\rho$.

Theorem 3.1. Assume that the identity component $G^{\circ}$ is reductive. Then a principal $G$-bundle $\mathcal{P}$ over the formal scheme $\widehat{U}$ admits an algebraization if and only if for a fixed exact representation $G \hookrightarrow G L(V)$ the associated vector bundle $\mathcal{P}_{V}$ admits an algebraization, i.e. satisfies the conditions of Corollary 2.12,

The "only if" part is obvious. Since by a result of Haboush, cf. Theorem 3.3 in [Ha1, the quotient $G L(V) / G$ is affine, the "if" part follows from the following general statement.

Proposition 3.2. Let $H$ be an affine algebraic group over $k$ and let $G$ be its closed subgroup such that $H / G$ is affine. Suppose that $\mathcal{P}$ is a principal $G$-bundle over $\widehat{U}$ such that the associated principal $H$-bundle $\mathcal{Q}=\mathcal{P}_{H}$ admits an algebraization. Then $\mathcal{P}$ admits an algebraization.

First we establish a preparatory result. As before, $U \subset X$ is an open subset satisfying $U \cap X_{0}=U_{0}$.

Lemma 3.3. Let $H$ be a linear algebraic group overe $k, Q$ be a principal $H$-bundle on $U$ and $\widehat{Q}$ be its completion. Also let $Y$ be an affine $H$-variety. Then for any section $\widehat{s}: \widehat{U} \rightarrow \widehat{Q}_{Y}$ there exists a section $s: W \rightarrow Q_{Y}$ on an open subset $W \subset U$ containing $U_{0}$, with completion equal to $\widehat{s}$. If $(W, s)$ and $\left(W^{\prime}, s^{\prime}\right)$ are two such algebraizations, then $s=s^{\prime}$ on $W \cap W^{\prime}$.

Proof. One can find an $H$-invariant linear subspace $V^{\vee} \subset k[Y]$ containing a set of generators of $k[Y]$ as a $k$-algebra. Then the surjection $S y m_{k}^{*}\left(V^{\vee}\right) \rightarrow k[Y]$ gives an $H$-equivariant closed embedding $Y \hookrightarrow V$ into the dual space $V$. This induces closed embeddings $Q_{Y} \hookrightarrow Q_{V}$ and $\widehat{Q}_{Y} \hookrightarrow \widehat{Q}_{V}$.

Therefore $\widehat{s}$ becomes a section of the vector bundle $\widehat{Q}_{V}$. By Proposition 2.11(ii) the completion of the coherent sheaf $i_{*} Q_{V}$ is isomorphic to $\widehat{i}_{*} \widehat{Q}_{V}$, and therefore by Proposition 2.4(i) there exists a unique section $\tilde{s}$ of $i_{*} Q_{V}$ with completion given by $\widehat{i}_{*} \widehat{s}$. Set $s=\left.\tilde{s}\right|_{U}$.

It remains to show that $s(W) \subset Q_{V}$ on some $W$ as above. Let $\mathcal{A}=\operatorname{Sym}^{*}\left(Q_{V}^{\vee}\right)$ be the sheaf of symmetric algebras on $U$ corresponding to $Q_{V}$ and $\mathcal{I} \subset \mathcal{A}$ be the 
ideal sheaf of $Q_{Y}$. The section $s$ gives the evaluation morphism $\rho: \mathcal{A} \rightarrow \mathcal{O}_{U}$. The sheaf $G=\rho(\mathcal{I})$ is coherent, being a subsheaf of $\mathcal{O}_{U}$. Since $\widehat{s}$ takes values in $\widehat{Q}_{Y}$, the completion $\widehat{G}$ is zero. By Corollary 10.8.12 in [EGAI this implies $\operatorname{Supp}(G) \cap U_{0}=\emptyset$; hence $W=U \backslash \operatorname{Supp}(G)$ satisfies the conditions of the lemma. The uniqueness of $s$ follows from the uniqueness of $\widetilde{s}$.

Proof of Proposition 3.2. Let $(U, Q)$ be an algebraization of $\mathcal{Q}$. In general, giving a principal $G$-bundle is equivalent to giving a principal $H$-bundle $\mathcal{R}$ together with a reduction to $G$, i.e. a section of the associated bundle $\mathcal{R}_{H / G}$ with the fiber $H / G$. Since $\mathcal{Q}$ is induced from $\mathcal{P}$, we get a section $\widehat{s}: \widehat{U} \rightarrow \mathcal{Q}_{H / G}$, and by the above lemma there exists $s: W \rightarrow Q_{H / G}$ such that $\widehat{s}$ is equal to its completion. Then $\mathcal{P}$ admits an algebraization $(W, P)$ where $P$ is the pullback of the principal $G$-bundle $Q \rightarrow Q_{H / G}$ via $s: W \rightarrow Q_{H / G}$.

\section{Categorical Formulations}

Proposition 4.1. The functor $\left.F \mapsto \widehat{F}\right|_{\widehat{U}}$ induces an eqivalence between the full subcategory of all coherent sheaves $E$ on $X$, which are locally free at the points of $U_{0} \subset X$ and have depth $h_{x} \geq 2$ at the points where $E$ is not locally free, and the full subcategory of locally free sheaves on $\widehat{U}$ admitting algebraization.

Proof. Let $(U, F)$ be an algebraization of $\mathcal{F}$. Then the sheaf $E=i_{*} F$ satisfies $E \simeq i_{*} i^{*} E$; hence by Proposition 2.4(iii) depth $E \geq 2$ for all $x \in Z=X \backslash$ $U$. We also observe that $E$ is uniquely determined by $\mathcal{F}$, since by Propositions 2.4(i) and 2.11(ii) it is the unique coherent sheaf on $X$ such that $\widehat{E} \simeq \widehat{i}_{*} \mathcal{F}$. Thus the functor described is essentially surjective on objects. For the morphisms, let $\mathcal{F}_{1}, \mathcal{F}_{2}$ be a pair of vector bundles on $\widehat{U}$ with algebraizations $\left(U, F_{1}\right)$ and $\left(U, F_{2}\right)$, respectively, which we may assume to be defined on the same $U$. Denote by $E_{1}=$ $i_{*} F_{1}, E_{2}=i_{*} F_{2}$ the corresponding coherent sheaves on $X$. Then $\operatorname{Hom}_{\widehat{U}}\left(\mathcal{F}_{1}, \mathcal{F}_{2}\right)=$ $\operatorname{Hom}_{\widehat{X}}\left(\widehat{i}_{*} \mathcal{F}_{1}, \widehat{i}_{*} \mathcal{F}_{2}\right)=\operatorname{Hom}_{X}\left(E_{1}, E_{2}\right)$, where the first equality is by adjunction of $i^{*}$ and $i_{*}$ and the second by Propositions 2.4(i) and 2.11(ii).

To formulate a result for principal bundles, let $\mathcal{B}\left(G, U_{0}\right)$ be the groupoid category in which the objects are given by pairs $(U, P)$, where $U \subset X$ is an open subset with $U \cap X_{0}=U_{0}$, and $P$ is a principal $G$-bundle on $U$. Morphisms from $(U, P)$ to $\left(U^{\prime}, P^{\prime}\right)$ are given by the set of equivalence classes of pairs $(W, \psi)$, where $W \subset U \cap U^{\prime}$ is an open subset with $W \cap X_{0}=U_{0}$ and $\psi:\left.\left.P\right|_{W} \rightarrow P^{\prime}\right|_{W}$ an isomorphism of $G$-bundles. Two such pairs $(W, \psi)$ and $\left(W, \psi^{\prime}\right)$ are equivalent if $\psi=\psi^{\prime}$ on $W \cap W^{\prime}$. Also denote by $\operatorname{Bun}(G, \widehat{U})$ the groupoid category of $G$-bundles on the formal scheme $\widehat{U}$. Completion along $U_{0}$ defines a functor $\Psi: \mathcal{B}\left(G, U_{0}\right) \rightarrow \operatorname{Bun}(G, \widehat{U})$. The following statement summarizes our results on algebraization of principal bundles.

Theorem 4.2. With the notation of Section 2.1,

(i) For any affine algebraic group $G$ over $k, \Psi: \mathcal{B}\left(G, U_{0}\right) \rightarrow \operatorname{Bun}(G, \widehat{U})$ is full and strict.

(ii) For $G=G L_{n}(k)$ the essential image of $\Psi$ is the full subcategory of rank $n$ vector bundles $\mathcal{F}=\varliminf_{i m} F_{p}$ on $\widehat{U}$ which satisfy the equivalent conditions (i)-(iii) of Corollary 2.12. 
(iii) Let $G \hookrightarrow H$ be a closed embedding of affine algebraic groups over $k$ such that $H / G$ is affine. Then the natural functor from $G$-bundles to $H$-bundles induces an equivalence of categories

$$
\mathcal{B}\left(G, U_{0}\right) \simeq \operatorname{Bun}(G, \widehat{U}) \times_{B u n(H, \widehat{U})} \mathcal{B}\left(H, U_{0}\right) .
$$

Proof. To prove (i) suppose that $\mathcal{P}, \mathcal{P}^{\prime}$ are two principal bundles on $\widehat{U}$ admitting algebraizations $P, P^{\prime}$, respectively, which we may assume to be defined on the same $U \subset X$. Let $\widehat{\psi}: \mathcal{P} \rightarrow \mathcal{P}^{\prime}$ be an isomorphism. We need to prove that there exists (perhaps after shrinking $U$ ) a unique isomorphism $\psi: P \rightarrow P^{\prime}$ with completion given by $\widehat{\psi}$. Let $\operatorname{Isom}\left(P, P^{\prime}\right)$ be the bundle of isomorphisms $P \rightarrow P^{\prime}$. Considering graphs of isomorphisms, we can identify $\operatorname{Isom}\left(P, P^{\prime}\right) \simeq G \backslash\left(P \times_{U} P^{\prime}\right)$. On the other hand, $P \times_{U} P^{\prime}$ is a principal bundle over $G \times_{k} G$. Define a left action of $G \times{ }_{k} G$ on $G$ by $(g, h) \cdot f=g f h^{-1}$; then $G \backslash\left(P \times_{U} P^{\prime}\right) \simeq\left(P \times_{U} P^{\prime}\right)_{G}$. Since $\widehat{\psi}$ gives a section $\widehat{s}$ of $\operatorname{Isom}\left(\mathcal{P}, \mathcal{P}^{\prime}\right)$, applying Lemma 3.3 to $H=G \times{ }_{k} G$ and $Y=G$, we get a unique algebraization $s:\left.W \rightarrow\left(P \times_{U} P^{\prime}\right)_{G} \simeq \operatorname{Isom}\left(P, P^{\prime}\right)\right|_{W}$, which corresponds to the required isomorphism $\psi$. This proves (i).

The statement of (ii) for objects holds by Corollary 2.12 and for morphisms by (i).

For (iii) first observe that the compositions $\mathcal{B}\left(G, U_{0}\right) \rightarrow \mathcal{B}\left(H, U_{0}\right) \rightarrow \operatorname{Bun}(H, \widehat{U})$ and $\mathcal{B}\left(G, U_{0}\right) \rightarrow \operatorname{Bun}(G, \widehat{U}) \rightarrow \operatorname{Bun}(H, \widehat{U})$ are canonically isomorphic; therefore one does get a functor

$$
\mathcal{B}\left(G, U_{0}\right) \rightarrow \operatorname{Bun}(G, \widehat{U}) \times_{B u n(H, \widehat{U})} \mathcal{B}\left(H, U_{0}\right) .
$$

On objects, this functor is an equivalence if for a $G$-bundle $\mathcal{P}$ on $\widehat{U}$, an $H$-bundle $Q$ on $U \subset X$ and an isomorphism $\phi: \mathcal{P}_{H} \simeq \widehat{Q}$, there exists an open subset $W \subset U$ with $W \cap X_{0}=U_{0}$, a $G$-bundle $P$ on $W$ and isomorphisms $\widehat{P} \simeq \mathcal{P}$ and $\left.P_{H} \simeq Q\right|_{W}$ which induce $\phi$ in a natural way. This is equivalent to finding an algebraization of the section $\widehat{s}: \widehat{U} \rightarrow \widehat{Q}_{H / G}$ induced by $\phi$, which was done in the proof of Proposition 3.2. On morphisms, without loss of generality it suffices to consider two $G$-bundles $P, P^{\prime}$ defined on the same open set $U$, and isomorphisms $\psi: P_{H} \simeq P_{H}^{\prime}, \widehat{\phi}: \widehat{P} \rightarrow \widehat{P}^{\prime}$ which have the same image in $\operatorname{Bun}(H, \widehat{U})$. We need to show that there exists a unique isomorphism $\phi: P \rightarrow P^{\prime}$ inducing $\widehat{\phi}$ and $\psi$ in the natural sense. But by (i) there exists a unique $\phi$ with completion equal to $\widehat{\phi}$. Since by assumption the isomorphisms $\psi^{\prime}=\phi_{H}$ and $\psi$ are equal after completion, $\psi^{\prime}=\psi$ by part (i). This finishes the proof.

\section{ACKNOWLEDGMENTS}

The author thanks V. Ginzburg who first formulated the problem of defining the Uhlenbeck functor and whose unpublished preprint on it (written jointly with the present author) served as a principal motivation for this work. Many thanks are also due to V. Drinfeld who conjectured the statement of Theorem 2.10(i), brought the author's attention to the references [SGA2], Ha1], and also suggested Example 2.9 in Section 2.2.

\section{REFERENCES}

[Ar] Artin, M.: Versal deformations and algebraic stacks, Invent. Math. 27 (1974), 165-189. MR0399094 (53:2945) 
[Ba] Baranovsky, V.: Bundles on non-proper schemes: representability, preprint arXiv:0810.0091.

[BFG] Braverman, A.; Finkelberg M.; Gaitsgory D.: Uhlenbeck spaces via affine Lie algebras, The unity of mathematics, 17-135, Progr. Math., 244, Birkhäuser, Boston, MA, 2006. MR:2181803(2007f:14008)

[DK] Donaldson, S. K.; Kronheimer, P. B.: "The geometry of four-manifolds". Oxford Mathematical Monographs. The Clarendon Press, Oxford University Press, New York, 1990. MR 1079726 (92a:57036)

[EGAI] Grothendieck, A.: EGA I "Le langage des schémas". Inst. Hautes Ètudes Sci. Publ. Math. No. 41960.

[EGAIII] Grothendieck, A.: EGA III "Ètude cohomologique des faisceaux cohérents, premiere partie". Inst. Hautes Ètudes Sci. Publ. Math. No. 111961.

[FGK] Finkelberg, M.; Gaitsgory, D.; Kuznetsov, A.: Uhlenbeck spaces for $A^{2}$ and the affine Lie algebra $\hat{s l}_{n}$. Publ. Res. Inst. Math. Sci. 39 (2003), no. 4, 721-766. MR2025461 (2005b:17051)

[Ha1] Haboush, W.J.: Homogeneous vector bundles and reductive subgroups of reductive algebraic groups, Amer. J. of Math. 100 (1978), no. 6, 1123-1137. MR522693 (80f:14007)

[Ha2] Hartshorne, R.: "Residues and Duality". Lecture Notes in Mathematics, No. 20 Springer-Verlag, Berlin-New York, 1966. MR0222093 (36:5145)

[Li] Li, J.: Algebraic geometric interpretation of Donaldson's polynomial invariants, Journal of differential geometry 37 (1993), 417-466. MR.1205451 (93m:14007)

[Lu] Lurie, J.: Ph.D. Thesis available through the MIT Library website.

[R] Raynaud, M.: "Théorèmes de Lefschetz en cohomologie cohérente et en cohomologie étale". Mém. Soc. Math. France, 41, Paris, 1975. MR0407021 (53:10804)

[SGA2] Grothendieck, A.: SGA 2 "Cohomologie locale des faisceaux cohérents et théorèmes de Lefschetz locaux et globaux". Revised reprint of the 1968 French original. Documents Mathématiques (Paris), 4. Société Mathématique de France, Paris, 2005.

Department of Mathematics, University of California - Irvine, Irvine, California 92697

E-mail address: vbaranov@math.uci.edu 